\title{
Universalità e particolarismo dei diritti fondamentali
}

\author{
Universalism and Particularism in Fundamental Rights
}

\author{
Giuseppe ZACCARIA \\ Università di Padova (Italia) \\ giuseppe.zaccaria@unipd.it
}

RECIBIDO: 26/09/2018 / ACEPTADO: 14/12/2018

\begin{abstract}
Riassunto: Vengono dapprima esaminate due posizioni universalistiche tra loro molto diverse: la teoria formale di Luigi Ferrajoli, di cui è mostrata la contraddittorietà rispetto alle premesse di un approccio avalutativo; e la teoria «sostanzialistica» di Francesco Viola, che rivendicando la doppia natura dei diritti fondamentali, come legal rights e come moral rights, presuppone che i diritti vengano positivizzati e perciò concretizzati nei processi interpretativi e applicativi. Successivamente sono analizzate criticamente due diverse tesi particolaristiche: quella di Luca Baccelli che, riconoscendo ai diritti una connotazione storica e culturale, ne afferma la genesi in particolari circostanze storiche; e quella di Gianluigi Palombella che lega i diritti fondamentali alla loro contingente funzione istituzionale. La conclusione del saggio sostiene la tesi dell'universalità dei diritti come incontro tra universale e particolare, sviluppato tramite un dialogo multiculturale che valorizzi le differenze in una logica più ampia.
\end{abstract}

Parole chiave: diritti fondamentali; universalità; particolarismo; diritto e morale.

\begin{abstract}
Despite the vast scientific production on the subject, the notion of fundamental rights is still controversial and problematic. The a. reviews some of the main positions of the current Italian legal-philosophical debate on fundamental rights. In the first part, two very different universalistic positions are examined: the formal theory of law of Luig Ferrajoli, of which the author shows the contradictions with respect to the premises of a value-free approach; and the «substantive» theory of law of Francesco Viola, who, claiming the double nature of fundamental rights - as legal rights and as moral rights - presupposes that rights are positivized and therefore concretized in the processes of interpretation and application of law. In the second part, the a. analyses critically two different particularistic approaches to rights: Luca Baccelli, recognizing the historical and cultural connotation of rights, finds their genesis in particular historical circumstances; while Gianluigi Palombella binds fundamental rights to their contingent institutional function. In the conclusion, the a. supports the thesis of the universality of rights as an encounter between universal and particular, developed through a multicultural dialogue that enhances differences in a broader logic.
\end{abstract}

Keywords: fundamental rights; universality; particularism; legal rights; moral rights. egli ultimi anni si è parlato, con sempre maggiore enfasi e diffusione, di diritti fondamentali. La letteratura in materia, sia in lingua italiana sia in ambito anglosassone, ma non solo, è aumentata enormemente, mentre l'espressione è divenuta una delle parole-chiave del lessico contempora- 
neo, «un discorso politico egemonico» ${ }^{1}$ addirittura abusato nella retorica corrente. E tuttavia, nonostante tale alluvionale produttività scientifica, nonostante l'intensità del dibattito e la forte incidenza dei diritti nella cultura e nella pratica giuridica contemporanea - tanto che il ragionare e il deliberare sui diritti è divenuto momento centrale della vita politica - la nozione di diritti fondamentali continua a restare assai spesso generica, indeterminata e controversa ${ }^{2}$, rimanendo non del tutto chiara l'esatta portata di simile qualificazione ed essendo la discussione caratterizzata da non pochi equivoci e fraintendimenti. Inoltre, all'inesauribile dilatazione del discorso sui diritti e alla loro proliferazione ${ }^{3}$ non ha corrisposto affatto nella pratica una sorta di marcia trionfale verso un loro rispetto ed una loro sempre più piena realizzazione: è un processo, piuttosto, doloroso e complesso che registra drammatiche sconfitte, palesi e laceranti violazioni e intrecciarsi di conflitti attorno ai diritti ${ }^{4}$. E' legittimo chiedersi se un'eccessiva inflazione dei diritti possa portare con sé una perdita del loro valore e minori possibilità di un loro reale rispetto.

Una prova eloquente del carattere controverso e problematico della nozione è rappresentata dalla estesa confusione e dalla frequente assenza di criteri analitici con cui spesso vengono usati indifferentemente termini come diritti fondamentali, diritti tout-court, diritti umani, diritti costituzionali, diritti inviolabili, principi. Inoltre, le espressioni di diritti umani (e della loro universalitàs) e di diritti fondamentali, benchè la loro storia lessicale e la loro area semantica non siano identiche, sono frequentemente usate come sovrapponibili ${ }^{6}$. Più in

1 Donnelly, J., Universal Human rights in Theory and Practice, Cornell University Press, Ithaca, NY, 2003, $2^{\mathrm{a}}$ ed., p. 38.

2 Sul punto, v. CAMPBELL, T., «Human Rights: A Culture of Controversy», Fournal of Law and Society, vol. 26 (1999), pp. 6-26; MAZZARESe, T., Diritti fondamentali, in Atlante di filosofia del diritto, vol. I, a cura di Pomarici U., Giappichelli, Torino, 2012, pp. 179-217.

3 Wellman, C., The Proliferation of Rights. Moral Progress or Empty Rhetoric, Westview Press, Boulder-Oxford, 1998; Trujlllo, I. e Viola, F., What Human Rights Are Not (or not Only). A Negative Path to Human Rights Practice, Nova Publishers, New York 2014, pp. 79 ss.

4 Bobbio, N., L'età dei diritti, Einaudi, Torino, 1990.

5 Sul tema dell'universalità dei diritti umani v. il classico saggio di KRIELE, M., «Zur Universalität der Menschenrechte, in Rechtssystem und praktische Vernunft», ed. R. Alexy e R. Dreier, $A R-$ SP-Beiheft, 51 (1993), pp. 47-61, trad. it. in Rivista internazionale di filosofia del diritto, 69 (1992), p. 3 ss. ; VIOLA, F., «L'universalità dei dirittti umani : un'analisi concettuale», in F. Botturi, F. Totaro, a cura di, Universalismo ed etica pubblica, in Annuario di Etica, 3 (2006), pp. 155-187; ID., «La controversa universalità dei diritti umani», in Studia Patavina, 64 (2017), pp. 235-251.

6 Così ad esempio da Rodotà, S., Repertorio di fine secolo, Laterza, Roma-Bari, 1999 (2 ed.), p. 3. Propone una distinzione tra diritti umani e diritti fondamentali PARIOTTI, E., I diritti umani. Tra giustizia e ordinamento giuridico, Utet, Torino, 2008, p. 5. Per Palombella, G., Dopo la 
particolare l'espressione diritti fondamentali viene usata tanto nel senso tecnicizzato di diritti costituzionalizzati, cioè configurati come tali nello Stato costituzionale democratico ${ }^{7}$, quanto in un senso teorico-generale o, se si vuole, assiologico-generico di diritti principali legati al valore della dignità umana, kantianamente assunta come fine e non come mezzo ${ }^{8}$. E' peraltro evidente come ogni diversa denominazione rifletta, ne sia consapevole o meno chi ne fa uso, diversi approcci e diversi lessici disciplinari, ma soprattutto opzioni teoriche tra loro diverse o addirittura alternative (e, ovviamente, possa portare con sé ben diverse forme di tutela). Ciò non stupisce perché la centralità teorica dei diritti fondamentali investe direttamente il concetto stesso di diritto e ne condiziona le diverse dimensioni, da quella ontologica a quella epistemologica, a quella fenomenologica9.

Per cercare pertanto di portare un po' di chiarezza e di distinzioni, di sciogliere qualche ambiguità in una materia ampia e ingarbugliata, la nostra ricognizione necessariamente rapida dovrà limitarsi all'ambito della filosofia del diritto italiana, dove peraltro si ritrovano puntualmente quei caratteri di indeterminatezza e di non univocità di cui abbiamo appena parlato sul piano generale.

Dopo la fine della Seconda Guerra Mondiale e la caduta dei totalitarismi, a partire dalla proclamazione della Dichiarazione universale del 1948, si inaugura nel secondo dopoguerra del Novecento quella che è stata definita da Norberto Bobbio come «l'età dei diritti» ${ }^{10}$. Si innesca, nell'evoluzione

certezza. Il diritto in equilibrio tra giustizia e democrazia, Dedalo, Bari, 2006, p. 26, si tratta di due modalità concettuali diverse in cui si può parlare di diritti.

7 E' questa, ad esempio, la posizione di ALEXY, R., Teoria dei diritti fondamentali, trad. it. di L. De Carlo,Il Mulino, Bologna 2012, che assume i diritti in quanto principi espressi dal Grundgesetz tedesco. Successivamente la prospettiva di Alexy subirà un'evoluzione radicale, nel senso di una fondazione dei diritti a partire dalle regole del discorso in «Diskurstheorie und Menschenrechte», in ID., Recht, Vernunft, Diskurs, Suhrkamp, Frankfurt a.M., 1995, pp. 127-164; e in «Diritti umani senza metafisica», Rivista internazionale di filosofia del diritto, n. 1 (2015), pp. 7-23.

8 Sul punto cfr. Dworkin, R., «Cosa sono i diritti umani», Ragion Pratica, 29 (2007), pp. 471; Ollero Tassara, A., Diritto «positivo» e diritti umani, Giappichelli, Torino,1998, p. 106.

9 In proposito, v. MAZZARESE, T., Prefazione a Neocostituzionalismo e tutela (sovra)nazionale dei diritti fondamentali, a cura di T. Mazzarese, Giappichelli, Torino, 2002, p. IX.

10 Bobbio, N., L'età dei diritti..., cit.; Henkin, L., The Age of Rights, Columbia University Press, New York, 1990. 
della vicenda storica dei diritti, che divengono un problema internazionale, un triplice processo di positivizzazione, di generalizzazione e internazionalizzazione, e infine di specificazione ${ }^{11}$, che ha condotto al riconoscimento, innovativo e dirompente, sia nel diritto interno che in quello internazionale, del diritto di ogni uomo e ogni donna ad avere diritti uguali e inalienabili, da far valere erga omnes, anche nei confronti degli Stati. Questo processo è simultaneo alla diffusione in Europa di costituzioni che presentavano significativi aspetti di novità rispetto ai documenti precedenti, contenendo articoli relativi ai diritti civili, sociali e politici dei cittadini, che divengono il fondamento e il criterio di legittimazione dell'ordine politico-sociale. Rispetto al passato c'è un rovesciamento radicale di prospettiva nel rapporto Stato-cittadini, sempre più considerato dal punto di vista dei diritti dei cittadini anziché da quello del sovrano. Gli esseri umani sono soggetti di diritti non soltanto entro i loro ordinamenti statali, ma anche nell'ordinamento internazionale e, se necessario, contro i loro Stati. Ciò corrisponde all'internazionalizzazione dei diritti fondamentali ${ }^{12}$, che divengono diritti sovrastatali, ai quali gli Stati si trovano vincolati, trattandosi di diritti riconosciuti non soltanto ai cittadini, ma alle persone a prescindere dalle loro diverse cittadinanze. Ma ciò corrisponde anche all'allargamento della sfera dei diritti, cui, per effetto di trasformazioni culturali profonde, si assiste negli stati costituzionali. La prima parte della Costituzione italiana, ad esempio, delinea un ordinamento basato sul valore della persona e sulla moltiplicazione dei diritti, assunti come pilastri portanti del nuovo edificio costituzionale. Attorno ai diritti si organizza e si definisce la nuova democrazia italiana, che tuttavia si trova a fare i conti, non sempre facili, con la loro centralità. Agli inizi degli anni Sessanta, l'intera Costituzione comincia ad essere percepita come giuridicamente vincolante e come documento non solo programmatico, ma che esige di essere realizzato (e non soltanto dal legislatore): via via si fa più chiaro che essa non rappresenta soltanto il gradino più alto nella gerarchia delle fonti del diritto, ma anche una norma fondamentale della convivenza civile, sociale e politica.

Nell'espressione diritti umani è evidente l'intento di connettere immediatamente alcuni diritti all'essere umano in quanto tale, rinviando ad aspetti

11 MazZarese, T., Diritti fondamentali..., cit., p. 195.

12 Sul tema v. ora il fascicolo monografico di «Diritto \& questioni pubbliche», XVII, 2017, n. 1, dedicato a $\ll$ Disordine delle fonti del diritto (inter)nazionale e tutela dei diritti fondamentali. 
fondamentali della vita individuale e collettiva ${ }^{13}$. Nella vicenda storica dei diritti in Occidente ${ }^{14}$, questi ultimi mantengono un rapporto privilegiato con i soggetti. La dialettica si muove tra i poli dell'eguaglianza e delle differenze: se per un verso i diritti paiono come inseparabili dall'eguaglianza - spettando a tutti gli esseri umani - per altro verso emerge la consapevolezza che le differenze non possono più essere messe tra parentesi, ma al contrario vanno considerate come aspetti significativi dell'essere umano. In primo piano emerge il rispetto per l'identità delle persone e per i loro diritti. Ma ciò che più interessa sottolineare è che se in una prima fase pare prevalere la tematica della definizione dei diritti e della loro universalità, in una seconda e successiva fase, che è poi quella della loro implementazione ${ }^{15}$, acquista sempre maggiore rilevanza il momento dell'applicazione dei diritti, che diviene sempre più cruciale ${ }^{16}$. L'individuazione stessa dei diritti fondamentali diviene un problema interpretativo ${ }^{17}$. A ciò corrisponde un ruolo sempre più nevralgico del giudice, che diviene l'interprete dei diritti fondamentali, colui che - riprendendo un elemento chiave del common law - li amministra e li difende, assumendo dunque un ruolo di primo piano nella realizzazione del progetto costituzionale o nell'implementazione delle carte internazionali; e in definitiva svolgendo una parte-chiave nell'evolversi del catalogo dei diritti. L'interpretazione del dettato normativo ad opera delle corti diviene parte integrante del diritto soggettivo, considerato come prodotto di un processo di concretizzazione.

Per parte sua la costituzione, in quanto atto costitutivo della convivenza civile, contiene e identifica i valori e i principi generali che debbono ispirare la produzione giuridica di ciascun determinato sistema giuridico. In tal modo i principi, riconosciuti nelle costituzioni ma anche nelle carte internazionali divengono un vincolo di contenuto per la normazione ordinaria. Questo

13 Pastore, B., Per un'ermeneutica dei diritti umani, Giappichelli, Torino, 2003, pp. 2-11; ID., «I diritti umani come ragioni», in ID., Decisioni, argomenti, controlli. Diritto positivo e filosofia del diritto, Giappichelli, Torino, 2015, p. 138.

14 Sulla quale v. il nostro «Epistemological and Ethical Implication of the Personalized Justice», in Conference: P5 Medicine and Fustice, a cura di S. D. Ferrara, Springer, 2018, nonchè CosTA, P., «Diritti fondamentali (storia)», in Enciclopedia del diritto-Annali, vol. II, Giuffrè, Milano, 2008, pp. 365-417.

15 Bоввіо, L'età dei diritti..., cit., pp. 15-16.

16 Viola, F., Diritti umani e globalizzazione del diritto, Scientifica, Napoli, 2009, p. 15.

17 PINO, G., Diritti e interpretazione. Il ragionamento giuridico nello Stato costituzionale, Il Mulino, 2010, p. 127. 
diritto di rango superiore detta il dover essere interno del diritto positivo ${ }^{18}$, ponendosi come vincolo contenutistico interno ai processi di positivizzazione e come limite ai sempre possibili arbitri e sopraffazioni del potere. Questo «dover essere» configura una «sfera dell'indecidibile» ${ }^{19}$, che nelle democrazie costituzionali è sottratta alle possibilità di modifica da parte di maggioranze politiche.

Se questo è il quadro generalissimo all'interno del quale si configura oggi la tematica dei diritti fondamentali, un sondaggio sintetico che si può effettuare entro il dibattito emerso negli ultimi anni in seno alla filosofia del diritto italiana ci può consentire di individuare alcune idee di fondo, rappresentative di posizioni più diffuse e articolate.

Una prima, basilare distinzione che si pone al centro del dibattito è quella tra concezioni universalistiche e concezioni particolaristiche dei diritti fondamentali. L'universalità, si sostiene da più parti ${ }^{20}$, è elemento concettuale essenziale della nozione di diritti. Apparentemente si tratta di una distinzione assai significativa. In realtà lo è molto meno ${ }^{21}$, se non si precisa il punto di vista preso in considerazione per parlare di universalismo o di particolarismo dei diritti fondamentali. Se non integriamo l'informazione precisando il concetto di universalità o di particolarità e l'idea di ciò che si ritiene siano i diritti fondamentali, la forza della distinzione sbiadisce e rischia di perdere consistenza.

Una prima, in certo senso esemplare prospettiva di universalità è quella di tipo formale, sostenuta da Luigi Ferrajoli in una serie di saggi pubblicati

18 Così Viola, F.; ZacCaria, G., Diritto e interpretazione. Lineamenti di teoria ermeneutica del diritto, Laterza, Roma-Bari, 2009 (6 ed.), p. 349.

19 Sul punto, v. Ferrajoli, L., Principia Iuris. Teoria del diritto e della democrazia, vol. 2, Laterza, Roma-Bari, 2007, p. 19, ma anche GARZON VALDES, E., Tolleranza, responsabilità e Stato di diritto. Saggi di filosofia morale e politica, a cura di P. Comanducci e T. Mazzarese, Il Mulino, Bologna, 2003, passim.

20 Così ad esempio KrIELE, M., «L'universalità dei diritti umani», Rivista internazionale di filosofia del diritto, 69 (1992), p. 3: «E' implicito nel concetto dei 'diritti dell'uomo' che li possiamo pensare solo come universali o che non possiamo pensarli affatto». Ma cfr. anche AYALA-LASSO, J., «The Universality of Human Rights», in D. Warner (ed.), Human Rights and Humanitarian Law: The Quest for Universality, Martinus Nijhoff, The Hague-Boston-London, 1997, pp. 87-94.

21 Cfr. Viola, F., «La controversa universalità dei diritti umani», Studia Patavina, cit., p. 235. 
nella rivista Teoria politica e condensati nel volume curato da Ermanno Vitale, che raccoglie il dibattito teorico nato dall'analisi di Ferrajoli ${ }^{22}$.

La definizione di diritti fondamentali proposta da Ferrajoli è una definizione teorica e puramente formale, che suona in questi termini: «sono ' diritti fondamentali' tutti quei diritti che spettano universalmente a 'tutti' gli esseri umani in quanto dotati dello status di persone, o di cittadini, o di persone capaci d'agire ${ }^{23}$. E' una definizione che, secondo le parole del suo stesso autore, vuol essere teorica e puramente formale. Teorica perché prescinde dalla circostanza di fatto che in questo o in quell'ordinamento i diritti fondamentali siano (o non siano) formulati in carte costituzionali o in leggi fondamentali e perfino dal fatto che siano (o non siano) enunciati in norme di diritto positivo. Puramente formale, nel senso che prescinde dalla natura degli interessi e dei bisogni tutelati con il loro riconoscimento quali diritti fondamentali, e si basa unicamente sul carattere universale della loro imputazione alla classe dei soggetti che ne sono titolari.

In definitiva la tesi di Ferrajoli si fonda su due elementi cardinali: da un lato l'universalità come connotato strutturale dei diritti fondamentali e dall'altro l'idea di una radicale differenza di struttura tra i diritti fondamentali, che in quanto universali sarebbero indisponibili, inalienabili e inviolabili, e i diritti patrimoniali, che in quanto particolari sarebbero invece disponibili, negoziabili e alienabili ${ }^{24}$.

Questa tesi di Ferrajoli, a prima vista attraente per la sua capacità di non legarsi ad un ordinamento specifico che preveda o non preveda il riconoscimento di diritti fondamentali, ha sollevato un ampio dibattito, cui hanno partecipato alcuni dei più autorevoli filosofi del diritto italiani (da Guastini a Jori a Zolo) ed è stata tuttavia oggetto di numerose critiche, che hanno investito diversi aspetti della formulazione di Ferrajoli, ma si sono appunto concentrate sui due elementi cardinali di cui abbiamo appena parlato, l'universalità e l'indisponibilità come tratti definitori dei diritti fondamentali: il primo attinente

22 Ferrajoli, L., «Diritti fondamentali», Teoria Politica, 14 (1998), n. 2, pp. 3-33; ID., «Diritti fondamentali nella teoria del diritto», Teoria Politica, 15 (1999), n. 1, pp. 49-92; ID., «I fondamenti dei diritti fondamentali», Teoria Politica, 16 (2000), n. 3, pp. 41-113; ID., Diritti fondamentali. Un dibattito teorico, a cura di E. Vitale, Laterza, Roma-Bari, 2008 (terza ed.). Una valutazione critica delle tesi di Ferrajoli è in MARCHETTONI, L., I diritti umani tra universalismo e particolarismo, Giappichelli, Torino, 2012, pp. 141 ss.

23 Ferrajoli, L., Diritti fondamentali..., cit., p. 5.

24 Sul punto v. anche L. Ferrajoli, L., «Principia iuris». Teoria del diritto e della democrazia, vol. 1, Laterza, Roma-Bari. 2007, pp. 724 ss., 759 ss. 
alla titolarità di tali diritti, il secondo come attinente alle modalità del suo esercizio.

Ha osservato ad esempio criticamente Riccardo Guastini che se l'universalità rappresenta agli occhi di Ferrajoli un elemento definitorio dei diritti fondamentali, nel senso che un diritto è fondamentale se ascritto a tutti, ad una classe universale di soggetti, questa stessa caratteristica si può benissimo ritrovare non soltanto in un diritto fondamentale, ma anche in un privilegio, che potrebbe anch'esso essere ascritto ad una classe universale di soggetti, al limite inclusiva di fatto di un solo membro ${ }^{25}$. $\mathrm{E}$ in certo senso infierisce nella critica Pietro Costa: «Niente ci impedisce di immaginare un ordinamento dove il diritto di fumare è un diritto fondamentale attribuito a tutti i soggetti maschi di età superiore agli ottant'anni» ${ }^{26}$. Aggiunge sempre criticamente Mario Jori che la distinzione operata da Ferrajoli tra diritti fondamentali (disponibili) e diritti patrimoniali (indisponibili) implica chiaramente una scelta di valore etico-politica non dichiarata, ma effettiva a favore dei diritti fondamentali, così contraddicendo l'idea di fondo di un'universalità concepita in modo avalutativo ed esclusivamente formale. In effetti l'obiettivo di Ferrajoli - la cui teoria vorrebbe essere rigorosamente non cognitivista - di fornire una definizione di diritti fondamentali indipendente da valori e perciò ideologicamente neutrale, fallisce, perché nel momento stesso in cui sostiene che la conseguenza «necessaria» di tale carattere formale sarebbe l'inalienabilità e l'indisponibilità degli interessi sostanziali in cui consistono i diritti fondamentali, egli introduce surrettizziamente degli elementi concernenti il contenuto dei diritti, dunque per nulla formali, e basati in ultima analisi su giudizi di valore $^{27}$. Anche una definizione di tipo formale può postulare un valore e così veicolare una concezione di tipo valutativo ${ }^{28}$.

Con la qualificazione di «universale» non ci si può riferire solamente all'estensione dei diritti (universalismo dei titolari), ma anche all'universalità dei contenuti dei diritti. La considerazione della titolarità è indissociabile dalla considerazione dei contenuti ${ }^{29}$. I due elementi van tenuti insieme congiuntamente ed esplicitamente, non surrettiziamente.

25 Guastini, R., «Tre problemi di definizione», in Ferrajoli, L., Diritti fondamentali..., cit., p. 45.

26 Costa, P., voce: Diritti fondamentali (storia)..., cit., p. 365.

27 Viola, F., L'universalità dei diritti umani..., cit., p. 169.

28 Così MaZZARese, T., Diritti fondamentali..., cit., p. 181.

29 Su questa linea anche GRIfFIN, J., On Human Rights, Oxford U.P., Oxford, 2008, su cui cfr. CrISP, R. (ed.), Griffin on Human Rights, Oxford U.P., Oxford, 2014. 
Se Ferrajoli sembra scambiare l'universalità con la generalità, del tutto diversa è la concezione di universalità dei diritti sviluppata da Francesco Vio$1 \mathrm{a}^{30}$, a riprova di quanto sopra sostenuto circa la scarsa significatività dell'espressione: i diritti umani (o fondamentali) sono universali.

$\mathrm{Al}$ contrario di Ferrajoli, Viola cerca una giustificazione «forte» dei diritti e della loro universalità, basata sul riconoscimento della dignità dell'essere umano, quale effetto del riconoscimento dell'umanità e della libertà dell'altro $^{31}$, distinto da noi ma in relazione con noi, e della sua kantiana irriducibilità a mero mezzo. Le diverse umanità scoprono una comunanza nella comune umanità.

L'itinerario di Viola è lungo e complesso. Prende le mosse dalla distinzione corrente, a sua volta ripresa dalle tesi di Ronald Dworkin ${ }^{32}$, tra moral rights, tendenzialmente universali, e legal rights, necessariamente particolari in ragione del carattere particolare della tutela legislativa e giurisdizionale ${ }^{33}$ per distinguere i diritti umani, intesi come pratica sociale, dai diritti naturali. Mentre questi ultimi sono connessi alla natura umana ed alla condizione naturale dell'essere umano, i diritti umani hanno una necessaria connessione strutturale con autorità, istituzioni e diritto positivo ${ }^{34}$. In tal modo i diritti sono «tecniche» per il riconoscimento e la protezione di scopi assiologici, dal momento che dipendono da un'etica dei valori retrostante e dai processi storici che li producono. In altre parole, i moral rights non sono diritti già formati, ma solo argomentazioni o giustificazioni che richiedono di essere riconosciute come legal rights. I primi considerano i diritti dal punto di vista della loro inerenza ad un soggetto, i secondi dal punto di vista della tutela

30 Oltre ai saggi L'universalità dei diritti umani: Un'analisi concettuale, Diritti umani e globalizzazione del diritto e La controversa universalità dei diritti umani sopra citati, sono anche da vedere Diritti dell'uomo diritto naturale etica contemporanea, Giappichelli, Torino, 1989, Etica e metaetica dei diritti umani, Giappichelli, Torino, 2000, nonché, con TrujILlo, I., What Human Rights Are Not (Or Not Only)..., cit.

31 Qui si avverte l'influsso di FINNIS, J.M., Legge naturale e diritti naturali, a cura di F. Viola, Giappichelli, Torino, 1996, pp. 223, 228, 239; sulla dignità umana come relational property, v. Luban, D., «The Rule of Law and Human Dignity: Re-examing Fuller's Canons», Hague Fournal on the Rule of Law, 2 (2010), pp. 29-47.

32 La distinzione di Dworkin è tra abstract rights e concrete rights: cfr. Taking Rights Seriously, Duckworth, London, 1978 (2 ed.), pp. 93-94; 98-100.

33 Viola, F., L'universalità dei diritti..., cit., p. 156.

34 VIOLA, F., «I diritti umani sono naturali?», in F. Botturi, R.Mordacci, a cura di, Natura in etica, in Annuario di Etica, 6 (2009), Vita e Pensiero, Milano, 2009, pp. 69-92; Trujillo, I.; Viola, F., What Human Rights..., cit., pp. 4-5. 
che quest'ultimo riceve da parte della società ${ }^{35}$, implicano una relazione con altri, con un contesto istituzionale. Nel caso di legal rights alla validità morale si aggiunge quella giuridico-positiva ${ }^{36}$ : il riconoscimento di un moral right come legal right esige l'attribuzione dei poteri adeguati a realizzarlo ${ }^{37}$. Qui Viola introduce un'importante precisazione: «I diritti sono universali quanto alla definizione e invece particolari quanto all'applicazione» ${ }^{38}$. Il fatto è che i diritti di cui parliamo sono diritti positivi: essi cioè nascono come tali solo quando iniziano ad essere riconosciuti in senso giuridico. Prima di allora sono solo istanze morali. Debbono essere positivizzati, ossia riconosciuti da autorità (internazionali, nazionali, o locali), produttive di diritto positivo, anche se il grado di positivizzazione può essere di diversa intensità ${ }^{39}$ : sin dal momento in cui si presentano in testi di soft law essi sono da considerare a tutti gli effetti diritto positivo. Bobbio chiama appunto «età dei diritti» quella in cui essi sono positivizzati, individuandone il punto di svolta nel passaggio dalla considerazione dell'uomo astratto a quella dell'uomo nelle diverse fasi della sua vita e nei suoi diversi stati. Il problema dell'universalità dei diritti va allora così riformulato: è possibile un'universalità di ciò che è positivo? La risposta è affermativa, giacchè la positivizzazione, che pure è particolarizzazione, non esclude di per sé la pretesa di universalità dei diritti perché lascia sempre un'eccedenza assiologica ed un margine per ulteriori evoluzioni e per nuovi diritti. Non è affatto contraddittorio affermare insieme l'universalità e il particolarismo dei diritti, giacchè la positivizzazione riflette situazioni di vita particolari. Tutte le positivizzazioni dei diritti non sono per definizione assolute, ma contingenti e implicano la necessità di un continuo «riaggiustamento» dei giudizi di valore nella fase applicativa dei diritti ai casi concreti. Nella concretezza degli ordinamenti infatti la valenza universale dei diritti va mediata con positivizzazioni che si realizzano con riferimento ad azioni concrete e individuali ${ }^{40}$.

E qui dobbiamo sottolineare una prima, rilevante novità dell'analisi di Viola rispetto alle precedenti (inclusa quella di Ferrajoli): poiché il concetto,

35 Pastore, B.; Viola, F.; Zaccaria, G., Le ragioni del diritto, Il Mulino, Bologna, 2017, p. 95.

36 Alexy, R., «Diritti umani senza metafisica», Rivista internazionale di filosofia del diritto, n. 1 (2015), p. 9.

37 Pastore, B.; Viola, F.; ZacCaria, G., Le ragioni del diritto..., cit., p. 95.

38 Viola, F., Diritti umani e globalizzazione..., cit., p. 14; ID., Etica e metaetica..., cit., pp. 93-94, 9798.

39 ID., L'universalità dei diritti..., cit, p. 156.

40 Pastore, B., Per un'ermeneutica dei diritti umani, Giappichelli, Torino, 2003, p. 171. 
di derivazione ricoeuriana ${ }^{41}$, di riconoscimento è strutturalmente coessenziale a quello di diritti, con riferimento ai diritti stessi entra necessariamente in gioco l'elemento della positivizzazione, e quest'ultimo porta inevitabilmente con sé il momento dell'interpretazione. I diritti esigono di essere concretizzati nei processi applicativi ${ }^{42}$ : e per far questo è necessario ricorrere non solo a dottrine politiche e morali, ma anche a giudizi empirici. Ancorchè universali, i diritti infatti non possono non essere particolarizzati e contestualizzati da regole che li positivizzino e tali regole esigono d'essere interpretate. Nell'assetto del nuovo costituzionalismo l'interpretazione delle regole è controllata dall'interpretazione dei valori-guida del sistema giuridico. Emergono a quest'altezza, dunque, la dimensione ermeneutica della positivizzazione dei diritti, la non univocità degli esiti dell'interpretazione nell'ipotesi in cui ne siano oggetto le disposizioni formulatrici di diritti fondamentali, nonché la tematica di possibili conflitti e di necessari bilanciamenti in presenza di più diritti fondamentali ${ }^{43}$.

Ma vi è anche un secondo aspetto basilare a caratterizzare la prospettiva di Viola. La condizione attuale del diritto contemporaneo - egli osserva - è contraddistinta da «pluralismo giuridico» (l'operare di norme di varia provenienza, anche esterna, in uno stesso ambito) ${ }^{44}$ e da «multiculturalismo» (la presenza in una società di molte culture diverse e l'apprezzamento della validità di tutte) ${ }^{45}$. Ma questi due elementi possono portare accanto alla valorizzazione delle differenze anche al disordine giuridico strutturale e al dissolvimento dell'idea di

41 Ricoeur, P., Sé come un altro, trad.it. di D. Iannotta, Jaka Book, Milano, 1993, pp. 275 ss., 290 ss. Su questi aspetti del pensiero di Ricoeur cfr. il nostro «Explicar y Comprender. En torno a la filosofia del derecho de Paul Ricoeur» Doxa, 22 (1999), pp. 631-641, nonché CANALE, D., «Ricoeur e la dialettica del riconoscimento», Rivista internazionale di filosofia del diritto, LXXVII (2000), pp. 292-322.

42 Opera una distinzione tra diritti fondamentali sull'applicazione giudiziale del diritto e nell'applicazione giudiziale del diritto, MAZZARESE, T., «Ancora su ragionamento giudiziale e diritti fondamentali. Spunti per una posizione politicamente scorretta», Ragion Pratica, 2 (2010), p. 521 ss.

43 Nell'amplissima bibliografia sul tema, v. almeno AleXY, R., A Theory of Constitutional Rights (1986), Oxford University Press, Oxford, 2002, cap. 3; MorEso, J.J., «Conflitti tra principi costituzionali», Ragion Pratica, 10, 18 (2002), pp. 201-221; ComanducCI, P., Problemi di compatibilità tra diritti fondamentali, in P. Comanducci, R. Guastini, a cura di, «Analisi e diritto» 20022003, Giappichelli, Torino, 2004, pp. 317-329; PINO, G., Diritti e interpretazione. Il ragionamento giuridico nello Stato costituzionale, Laterza, Roma-Bari, 2010, pp. 143-199; GUASTINI, R., Saggi scettici sull'interpretazione, Giappichelli, Torino, 2017, pp. 97 ss.

44 Griffith, J., «What is Legal Pluralism?», Legal Pluralism \& Unofficial Law, 24 (1986), p. 38 ss.; VIOLA, F., «Ermeneutica filosofica, pluralismo e diritto», Etica \& Politica, 1 (2006), pp. 1-19.

45 PARIOTTI, E., «Multiculturalismo, globalizzazione e universalità dei diritti umani», Ragion Pratica, 9,16 (2001), pp. 63-86. 
un sistema giuridico da un lato, e alla conseguenza di un radicale relativismo culturale dall'altro. Qui Viola recupera al diritto un ruolo essenziale di riordino e di integrazione delle differenze: se infatti i problemi della vita comune sono effettivamente comuni, cioè ricorrenti in tutte le società, universalità dei diritti non significa necessariamente loro assolutezza, ma piuttosto comunanza, cioè capacità di accomunare cose diverse, modi di intendere diversi, il che richiede la produzione di argomenti e di ragioni. Se i diritti umani sono universali, allora diviene plausibile la ricerca di una loro giustificazione nella comune umanità ${ }^{46}$. La comunanza nel ragionamento giuridico rende possibile la comunanza di norme e istituti giuridici, pur nella pluralità dei modi di concepire i valori fondamentali e di interpretare il carattere controverso del contenuto dei diritti, nella quale giocano senza dubbio un ruolo importante le condizioni di praticabilità e di esercizio dei diritti nelle concrete situazioni storiche. Ciò che è umano, perché è comune a tutti, va riconosciuto e assicurato a tutti gli esseri umani pur nella singolarità degli eventi e dei contesti. Dunque, il concetto di universalità non richiede di necessità quello di assolutezza (che renderebbe impossibile la relazione tra culture diverse) e può permettere l'instaurarsi di relazioni, a patto che le ragioni giustificative dei diritti siano universali.

Insomma, non bisogna confondere l'universalismo normativo con l'assolutismo morale, che nega la possibilità di una realizzazione dell'universale nel particolare in una pluralità di modi ${ }^{47}$.

Se i diritti valgono per tutti in quanto comuni a tutti, essi però - come abbiamo appena visto - si realizzano come particolari (hanno cioè bisogno di essere contestualizzati in situazioni reali), non come particolaristici, perché in questi ultimi, a differenza di quanto avviene nei diritti particolari, non è presente l'elemento della comunanza, il fattore accomunante, che è poi l'elemento decisivo per sostenere che i diritti debbono valere per tutti ${ }^{48}$.

A quest'altezza si incrocia una delle obiezioni più diffuse e ricorrenti all'universalità dei diritti ${ }^{49}$, quella che ne afferma il particolarismo, sostenendo ad

46 Viola, F., «Introduction: Natural Law Theories in the 20th Century», in Legal Philosophy in the Twentieth Century: The Civil Law World, t. 2, ed. E. Pattaro, C. Roversi, Springer, Meppel, The Netherlands, 2016, p. 88.

47 ViOLA, F., La controversa universalità..., cit., p. 235.

48 O'NeILl, O., Towwards fustice and Virtue: A Constructive Account of Practical Reasoning, Cambridge U. P., Cambridge, 1996, p. 74.

49 Per un'efficace sintesi delle varie obiezioni alla convinzione dell'universalità dei diritti, v. VIOLA, F., La controversa universalità..., cit., p. 241 ss. 
esempio che i diritti derivano dalle tradizioni culturali di specifiche aree geografiche o che essi non sono che il frutto dell'ideologia occidentale ${ }^{50}$, in contraddizione con il rispetto dovuto a soggetti e popoli di cultura diversa ai quali si pretenderebbe di imporli. O addirittura che costituiscono un'ideologia che legittima l'imperialismo ${ }^{51}$ dei valori occidentali, ossia mire egemoniche ed interferenze indebite nelle scelte delle comunità politiche. Il contenuto dei diritti sarebbe pertanto determinato dalle pratiche contingenti delle diverse società.

Un'articolazione paradigmatica di simili tesi, che pongono in modo critico la questione dell'universalizzazione dei diritti a livello globale e sottolineano il particolarismo sotteso al linguaggio universalistico dei diritti - giacchè a proporre i criteri universali sono sempre soggetti particolari - si trova nella prospettiva di Luca Baccelli, in particolare nel saggio I diritti dei popoli. Universalismo e differenze culturali ${ }^{52}$. Per sostenere la tesi che l'universalismo dei diritti umani non è filosoficamente fondato e in sostanza per prendere congedo dall'universalismo ${ }^{53}$, Baccelli muove dalla convinzione che l'origine del concetto di diritti soggettivi, che sta alla base dell'idea occidentale di diritti, pur caratterizzata da una genealogia assai complessa, sia contraddistinta dal particolarismo (partendo dall'affermazione particolaristica di immunità, franchigie e privilegi) e divenga significativa soprattutto nel mondo moderno, nel quale viene posto al centro il tentativo di definirne i diversi significati.

La rivendicazione di determinati diritti, la «lotta per i diritti» nasce storicamente collocata ed attiva un movimento dal particolarismo all'universalizzazione $^{54}$ : per dirla con le parole dello stesso Baccelli, «se i diritti esprimono

50 La questione fu posta da R. PANNIKaR, «La notion des droits de l'homme est-elle un concept occidental?», Diogene, 120 (1982), pp. 87-115.

51 Milne, A. J. M., Human Rights and Human Diversity, State University of New York Press, Albany, 1986; ZolO, D., Cosmopolis. La prospettiva del governo mondiale, Feltrinelli, Milano, 1995; ID., Tramonto globale. La fame, il patibolo, la guerra, Firenze University Press, Firenze, 2010; MARCHETTONI, I diritti umani tra universalismo..., cit., p. 175

52 Laterza, Roma-Bari, 2009, ma v. anche Il particolarismo dei diritti. Poteri degli individui e paradossi dell'universalismo, Carocci, Roma, 1999, su cui cfr. la nota di MAZZARESE, T., «Diritti fondamentali tra particolarismo e universalismo», Ragion Pratica, IX (2001), n. 17, pp. 205-213.

53 BACCELLI, Il particolarismo dei diritti..., cit., p. 145.

54 ID., I diritti dei popoli..., cit., p. 98. 
pratiche sociali, non vi è alcun bisogno di ricercare universali antropologici cui debbano essere riferiti o inseguire categorie trascendentali. I differenti diritti emergono in particolari condizioni storiche come risposta di determinati soggetti a specifiche situazioni di violenza, di oppressione, di dominio, di misconoscimento, di discriminazione ${ }^{55}$. Dunque, ai diritti umani viene riconosciuta una connotazione storica e culturale, prendendo sul serio le differenze culturali e proponendo come desiderabile un confronto interculturale che favorisca l'allargamento della base di accettazione dei diritti umani e la «traduzione» dei valori che riflettono culture diverse. Il tutto mantenendo ferma la consapevolezza che al di sotto del dialogo interculturale permangono rapporti di potere e di violenza ${ }^{56}$.

La prospettiva di Baccelli coglie indubbiamente un punto non secondario: nella cultura occidentale il linguaggio e la pratica dei diritti hanno adempiuto una funzione importante, volta a formulare rivendicazioni e a lottare per una tutela dalle discriminazioni e dall'oppressione. Ma anche se si può legittimamente ipotizzare che essi possano rappresentare una sorta di «codice di convivenza» in contesti in cui convivono identità diverse ed in cui differenti soggetti culturali rivendicano il riconoscimento della propria identità ${ }^{57}$, resta pur vero che ammettendo che diverse culture particolari comunichino tra loro, si deve presupporre che tra loro intercorra qualcosa di comune ${ }^{58}$. Non solo: presupponendo, pur a partire da posizioni particolaristiche, di attribuire un eguale valore alle diverse identità culturali non si rischia di operare una critica dell'universalismo in nome di quello stesso universalismo che si intende contestare ${ }^{59}$ ?

Probabilmente, allora, più che contrapporre un po' astrattamente universalismo e particolarismo, è più fruttuoso immaginare forme diverse di collegamento tra il polo dell'universalità e quello della particolarità. E' vero che le istanze particolaristiche possono mettere in moto un processo di universalizzazione - come la storia delle rivendicazioni dei diritti in origine legata all'antropologia liberale e all'individualismo dimostra - ma è altrettanto vero che questo non sarebbe possibile prescindendo dalla forza della giustificazione

55 Ibid., p. 122. Qui le tesi di Baccelli sono influenzate da BobBIo, L'età dei diritti..., cit.

56 Ibid., p. 116.

$57 \mathrm{Al}$ riguardo cfr. BELVISI, F., «I diritti fondamentali nelle società multiculturali», Diritto e società, n. 1 (2012), pp. 1-18.

58 VIOLA, F., La controversa universalità..., cit., p. 250.

59 E'la critica che a Baccelli rivolge FerRajoli, Diritti fondamentali..., cit., pp. 340-341. 
normativa dei diritti, che, come ogni opera della ragione, tende all'universalità ${ }^{60}$. Negare l'universalità dei diritti implicherebbe negarne la validità in quanto criteri assiologici, e perdere così un elemento concettuale essenziale dei diritti stessi, il loro senso normativo. Il che, nell'ambito delle organizzazioni giuridiche, significherebbe inevitabilmente anche perderne la sovraordinazione normativa (il fatto di essere posti come fini superiori) e la priorità all'interno del diritto positivo.

Possono esservi vie diverse per sostenere la tesi dell'universalità dei diritti: l'incontro tra l'universale e il particolare può avvenire muovendo dall'alto o dal basso. La via che parte dall'alto è oggi la più criticata, giacchè si afferma che l'idea di un fondamento ultimo, consistente nella comune natura umana, non è sostenibile in un mondo pluralistico e multiculturale come quello attuale. Solo in apparenza alternativa la via che muove dal basso, di diritti collocati in precisi contesti culturali, per volgersi alla ricerca dell'universale, che diviene a sua volta un nuovo punto di partenza per una considerazione più piena e ricca dell'universalità. Le due strade per riconoscere l'universalità dei diritti sono in realtà complementari. Allora l'universalità diviene il risultato di un processo che si sviluppa tramite tentativi e confronti, tramite un dialogo interculturale che non assolutizza alcun modello, pur assumendone alcuni come principali, e che si mostra capace di far dialogare le diversità e insieme di sussumerne il particolarismo in una logica più ampia. Potranno pure essere parzialmente inefficaci le proclamazioni giuridiche dei diritti, ma esse mantengono la funzione benefica di orientare e stimolare l'azione sociale e politica, pur non essendo ancora pienamente reali le condizioni per dare soddisfazione ed effettività ai diritti stessi ${ }^{61}$.

L'ordine giuridico è caratterizzato da una progressiva determinatez$\mathrm{za}$, da un passaggio continuo dall'indeterminato al determinato. In questo movimento della ragione pratica emerge il nesso valori-principi-norme $e^{62}$, che diviene l'articolata sequenza per cui le istanze assiologiche, i valori che avanzano pretese di giustizia e che danno un orientamento all'esistenza si

60 VIOLA, F., La controversa universalità..., cit., p. 240.

61 Sul carattere dinamico del processo di giustificazione dei diritti umani, cfr. BUCHANAN, A., «Diritti umani: i limiti del ragionamento filosofico», Ragion Pratica, 1 (2009), p. 33.

62 Sul punto, cfr. Alexy, R., «On Necessary Relations Between Law and Morality», Ratio furis, 2, 2 (1989), pp. 167-183, ma anche VIOLA, F., «Diritti umani e ragion pratica», Metodo. International Studies on Phenomenology and Philosophy, 2, 2, 1 (2014), pp. 49 ss.; ID., La controversa universalità..., cit., p. 47. 
trasformano in principi giuridici e infine si articolano nel linguaggio delle norme, che attribuiscono poteri giuridici e impongono correlativi doveri, per garantire la tutela e il rispetto dei diritti. La positivizzazione dei diritti richiede un costante impegno ermeneutico ed esercizio del giudizio in situazioni concrete che perviene alla determinazione di concrete forme di protezione in specifici contesti.

\section{5.}

Abbiamo insistito, nel corso di questo nostro contributo, sul carattere interpretativo dei diritti, che per essere storicamente praticabili debbono essere riconosciuti e accolti dal punto di vista del diritto positivo. Questo implica che essi siano assunti in una forma compatibile con i fini collettivi, o, se si vuole, che siano effettivi in un ordinamento giuridico concreto. Di qui la tesi - sostenuta da Gianluigi Palombella - che i diritti entrano a far parte dell'assetto istituzionale di una società come meta-norme fondamentali di tipo «sostantivo» ${ }^{63}$. In altre parole i diritti non sono solo una questione individuale, inerente alle singole persone, ma anche una questione istituzionale.

Nella teoria funzionale di Palombella ${ }^{64}$ - in questo debitrice della concezione di Luhmann ${ }^{65}$, alla quale tuttavia non è completamente riducibile -ciò che rende fondamentali i diritti è per l'appunto la loro funzione: più in particolare essi possiedono una rilevante qualità costitutiva dell'ordine giuridico, sono cioè produttivi di autorità. Si tratta di una prospettiva giuspositivistica che, rovesciando l'ottica «individualistica» con cui spesso ci si è accostati ai diritti fondamentali, li considera come giuridicamente vincolanti. Essi funzionano come «norme di riconoscimento» in un dato ordinamento. Come si vede, le tesi di Palombella riferiscono i diritti a ciò che in un ordinamento può valere come fondamentale: è un'ottica che eleva i diritti fondamentali a criteri di legittimità su cui si basa il sistema giuridico e politico e che perciò non accetta di separare il tema dei diritti dalla costruzione politica e istituzionale. In questo essa si rivela implicitamente polemica nei confronti di molti approcci

63 Palombella, Dopo la certezza..., cit., p. 41.

64 Esposta soprattutto in L'autorità dei diritti. I diritti fondamentali tra istituzioni e norme, Laterza, Roma-Bari, 2002.

65 Luhmann, L., I diritti fondamentali come istituzione, a cura di G. Palombella e L. Pannarale, Dedalo, Bari, 2002. 
contemporanei al problema dei diritti - da quello di James Griffin ${ }^{66}$ a quello di Amartya $\mathrm{Sen}^{67}$ ad esempio - che non prendono in considerazione il ruolo delle istituzioni riguardo ai diritti umani.

Ma legare strettamente i diritti allo Stato e renderli dipendenti dall'insieme delle istituzioni pubbliche comporta inevitabilmente la perdita di universalità. Infatti se i diritti sono istituzioni, sono istituzioni sociali che si consolidano e si sviluppano in una precisa fase dell'evoluzione sociale e a questa legano il proprio destino. Se si vuole stabilire quali tra i diritti siano e possano dirsi veramente fondamentali, non si può che rifarsi ad un'antropologia della persona che veda nella dignità di quest'ultima ${ }^{68}$ un carattere inalienabile, inviolabile e indisponibile.

In conclusione, pur esigendo di essere contestualizzati e dovendo costantemente commisurarsi con determinazioni particolari e dunque con concretizzazioni ed autocorrezioni, i diritti umani, in quanto spettanze dovute ad ogni essere umano in quanto tale, sono giustificabili erga omnes, travalicano i poteri che li riconoscono, possiedono un valore intrinseco ed una tensione strutturale all'indisponibilità, e rivelano una portata universale necessariamente più ampia di quella delle comunità politiche in cui si contestualizzano.

\section{REFERENCIAS}

AleXY, R., «On Necessary Relations Between Law and Morality», Ratio furis, 2, 2 (1989), pp. 167-183.

- «Diskurstheorie und Menschenrechte», in ID., Recht, Vernunft, Diskurs, Suhrkamp, Frankfurt a.M., 1995, pp. 127-164.

- A Theory of Constitutional Rights, Oxford University Press, Oxford, 2002.

- Teoria dei diritti fondamentali, trad. it. di L. De Carlo,Il Mulino, Bologna 2012.

66 Griffin, J., «Discrepancies between the Best Philosophical Account of Human Rights and the International Law of Human Rights», Proceedings of the Aristotelian Society, 101 (2001), 2.

67 SEN, A., «Elements of Theory of Human Rights», in Philosophy \& Public Affairs, 32, 4 (2004), pp. 315-356.

68 Sul tema cfr. Spaemann, R., «Sobre el concepto de dignidad humana», Persona y Derecho, 19 (1988), pp. 14-33; ID., Persone. Sulla differenza tra «qualcosa» e «qualcuno», a cura di R. Allodi, Laterza, Roma-Bari, 2007; FINNIS, J., «The Priority of Persons» (2000), in Collected Essays, vol. II, Oxford U.P., Oxford 2011, pp. 19-35; Waldron, J., Dignity, Rank und Rights, Oxford U. P., Oxford, 2012; Ciaramelli, F. (a cura di), «La dignità delle persone: storia e teorie», Rivista di filosofia del diritto, 2, 2 (2013), pp. 281-333. 
- «Diritti umani senza metafisica», in Rivista internazionale di filosofia del diritto, n. 1 (2015), pp. 23-36.

Ayala-Lasso, J., «The Universality of Human Rights», in D. Warner (ed.), Human Rights and Humanitarian Law: The Quest for Universality, Martinus Nijhoff, The Hague-Boston-London, 1997, pp. 87-91.

BaCELLI, L., Il particolarismo dei diritti. Poteri degli individui e paradossi dell'universalismo, Carocci, Roma, 1999.

- I diritti dei popoli. Universalismo e differenze culturali, Laterza, Roma-Bari, 2009.

BELVISI, F., «I diritti fondamentali nelle società multiculturali», Diritto e società, n. 1 (2012), pp. $1-18$.

BobBIO, N., L'età dei diritti, Einaudi, Torino, 1990.

Buchanan, A., «Diritti umani: i limiti del ragionamento filosofico», Ragion Pratica, 1 (2009), pp. 29-64.

Campbell, T., «Human Rights: A Culture of Controversy», Fournal of Law and Society, vol. 26 (1999), pp. 6-26.

CANALE, D., «Ricoeur e la dialettica del riconoscimento», Rivista internazionale di filosofia del diritto, LXXVII (2000), pp. 292-322.

Ciaramelli, F. (a cura di), «La dignità delle persone: storia e teorie», Rivista di filosofia del diritto, 2, 2 (2013), pp. 321-334.

Comanducci, P., «Problemi di compatibilità tra diritti fondamentali», in P. Comanducci, R. Guastini, a cura di, Analisi e diritto, 2002-2003, Giappichelli, Torino, 2004, pp. 317-329.

Costa, P., «Diritti fondamentali (storia)», in Enciclopedia del diritto-Annali, vol. II, Giuffrè, Milano, 2008, pp. 365-417.

Crisp, R. (ed.), Griffin on Human Rights, Oxford U.P., Oxford, 2014.

Donnelly, J., Universal Human rights in Theory and Practice, Cornell University Press, Ithaca, NY, 2003, $2^{\mathrm{a}}$ ed.

DwOrkin, R., «Cosa sono i diritti umani», Ragion Pratica, 29 (2007), pp. 469-482.

FErrajoli, L., «Diritti fondamentali», Teoria Politica, 14 (1998), pp. 3-33.

— «Diritti fondamentali nella teoria del diritto», Teoria Politica, 15 (1999), pp. 49-92.

— «I fondamenti dei diritti fondamentali», Teoria Politica, 16 (2000), pp. 41-114.

- «Principia iuris». Teoria del diritto e della democrazia, vols. 1-2, Laterza, Roma-Bari. 2007.

- Diritti fondamentali. Un dibattito teorico, a cura di E. Vitale, Laterza, Roma-Bari, 2008 (terza ed.).

FINNIS, J. Legge naturale e diritti naturali, a cura di F. Viola, Giappichelli, Torino, 1996.

— «The Priority of Persons» (2000), in Collected Essays, vol. II, Oxford U. P., Oxford 2011, pp. 19-35.

Garzon VAldes, E., Tolleranza, responsabilità e Stato di diritto. Saggi di filosofia morale e politica, a cura di P. Comanducci e T. Mazzarese, Il Mulino, Bologna, 2003.

GrIfFIN, J., «Discrepancies between the Best Philosophical Account of Human Rights and the International Law of Human Rights», Proceedings of the Aristotelian Society, 101 (2001), pp. 1-28.

- On Human Rights, Oxford U.P., Oxford, 2008. 
GrIfFITH, J., «What is Legal Pluralism?», Legal Pluralism \& Unofficial Law, 24 (1986), pp. $1-55$.

GuASTINI, R., «Tre problemi di definizione», in L. Ferrajoli, Diritti fondamentali. Un dibattito teorico, a cura di E. Vitale, Laterza, Roma-Bari, 2008 (terza ed.), pp. 43-48.

- Saggi scettici sull'interpretazione, Giappichelli, Torino, 2017.

Henkin, L., The Age of Rights, Columbia University Press, New York, 1990.

KRIELE, M., «L'universalità dei diritti umani», Rivista internazionale di filosofia del diritto, 69 (1992), pp. 3-26.

Luban, D., «The Rule of Law and Human Dignity: Re-examing Fuller's Canons», Hague Fournal on the Rule of Law, 2 (2010), pp. 29-47.

LuHmann, N., I diritti fondamentali come istituzione, a cura di G. Palombella e L. Pannarale, Dedalo, Bari, 2002.

MARCHETTONI, L., I diritti umani tra universalismo e particolarismo, Giappichelli, Torino, 2012.

MAZZARESE, T., «Diritti fondamentali tra particolarismo e universalismo», Ragion Pratica, 9, 17 (2001), pp. 205-213.

- Prefazione a Neocostituzionalismo e tutela (sovra)nazionale dei diritti fondamentali, a cura di T. Mazzarese, Giappichelli, Torino, 2002.

- «Ancora su ragionamento giudiziale e diritti fondamentali. Spunti per una posizione politicamente scorretta», Ragion pratica, 35 (2010), pp. 509-548.

- «Diritti fondamentali», in Atlante di filosofia del diritto, vol. I, a cura di Pomarici U., Giappichelli, Torino, 2012, pp. 179-217.

Milne, A.J.M., Human Rights and Human Diversity, State University of New York Press, Albany, 1986.

Moreso, J.J., «Conflitti tra principi costituzionali», in Ragion Pratica, 10, 18 (2002), pp. 201-221.

O’NeILl, O., Towards Justice and Virtue: A Constructive Account of Practical Reasoning, Cambridge U.P., Cambridge, 1996.

Ollero Tassara, A., Diritto «positivo» e diritti umani, Giappichelli, Torino, 1998.

Palombella, G., Dopo la certezza. Il diritto in equilibrio tra giustizia e democrazia, Dedalo, Bari, 2006.

PANNIKAR, R., «La notion des droits de l'homme est-elle un concept occidental?», in Diogene, 120 (1982), pp. 87-115.

PARIOTTI, E., «Multiculturalismo, globalizzazione e universalità dei diritti umani», in Ragion pratica, 9, 16 (2001), pp. 63-85.

- I diritti umani. Tra giustizia e ordinamento giuridico, Utet, Torino, 2008.

Pastore, B., Per un'ermeneutica dei diritti umani, Giappichelli, Torino, 2003.

- «I diritti umani come ragioni», in ID., Decisioni, argomenti, controlli. Diritto positivo e filosofia del diritto, Giappichelli, Torino, 2015, pp. 113-138.

Pastore, B.; Viola, F.; ZacCARIa, G., Le ragioni del diritto, Il Mulino, Bologna, 2017.

PINO, G., Diritti e interpretazione. Il ragionamento giuridico nello Stato costituzionale, Laterza, Roma-Bari, 2010.

Ricoeur, P., Sé come un altro, trad. it. di D. Iannotta, Jaka Book, Milano, 1993.

RoDotà, S., Repertorio di fine secolo, Laterza, Roma-Bari, 1999 (2 ed.). 
SEN, A., «Elements of Theory of Human Rights», Philosophy \& Public Affairs, 32, 4 (2004), pp. 315-356.

Spatmann, R., «Sobre el concepto de dignidad humana», Persona y Derecho, 19 (1988), pp. 14-33.

- Persone. Sulla differenza tra «qualcosa» e «qualcuno», a cura di R. Allodi, Laterza, Roma-Bari, 2007.

Trujillo, I.; Viola, F., What Human Rights Are Not (or not Only). A Negative Path to Human Rights Practice, Nova Publishers, New York 2014.

Viola, F., «Ermeneutica filosofica, pluralismo e diritto», Etica \& Politica, 1 (2006), pp. $1-20$.

- «L'universalità dei dirittti umani: un'analisi concettuale», in F. Botturi, F. Totaro, a cura di, «Universalismo ed etica pubblica», in Annuario di Etica, 3 (2006), pp. 155 187.

- Diritti umani e globalizzazione del diritto, Editoriale Scientifica, Napoli, 2009.

- «I diritti umani sono naturali?», in F. Botturi, R. Mordacci, a cura di, «Natura in etica», Annuario di Etica, 6 (2009), pp. 65-92.

- Diritti umani e ragion pratica, in «Metodo. International Studies on Phenomenology and Philosophy» 2, 2, 1 (2014), pp. 49-58.

- Introduction: Natural Law Theories in the 20th Century, in Legal Philosophy in the Twentieth Century: The Civil Law World, t. 2, E. Pattaro, C. Roversi (ed.), Springer, Meppel, The Netherlands, 2016, pp. 3-90.

— «La controversa universalità dei diritti umani», Studia Patavina, 64 (2017), pp. 235 251.

VIOLA, F.; ZACCARIA, G., Diritto e interpretazione. Lineamenti di teoria ermeneutica del diritto, Laterza, Roma-Bari, 2009 (6 ed.).

Waldron, J., Dignity, Rank und Rights, Oxford U. P., Oxford, 2012.

Wellman, C., The Proliferation of Rights. Moral Progress or Empty Rhetoric, Westview Press, Boulder-Oxford, 1998.

ZacCARIA, G., «Explicar y Comprender. En torno a la filosofia del derecho de Paul Ricoeur», Doxa, 22 (1999), pp. 631-641.

- «Epistemological and Ethical Implication of the Personalized Justice», in Conference: P5 Medicine and Fustice, a cura di S. D. Ferrara, Springer, 2018, pp. 37-41.

Zolo, D., Cosmopolis. La prospettiva del governo mondiale, Feltrinelli, Milano, 1995.

- Tramonto globale. La fame, il patibolo, la guerra, Firenze University Press, Firenze, 2010. 